Research Article

\title{
Theory of Frequency Captured of Eccentric Rotor by Vibration Environment with Same Direction
}

\author{
Su Ming (D), Li Rong, Xie Zhiping, and Zheng Jiming \\ School of Mechanical \& Electrical Engineering, Guizhou Normal University, Guiyang 550025, China \\ Correspondence should be addressed to Su Ming; suming629@163.com
}

Received 15 August 2020; Revised 4 December 2020; Accepted 30 December 2020; Published 20 January 2021

Academic Editor: N. A. Saeed

Copyright (c) 2021 Su Ming et al. This is an open access article distributed under the Creative Commons Attribution License, which permits unrestricted use, distribution, and reproduction in any medium, provided the original work is properly cited.

\begin{abstract}
Aiming at the frequency synchronization phenomena of oscillating or rotating bodies, this paper proposes a novel solution to address the self-synchronization problem of vibration systems. An integral mean method with small parameters and periodic coefficient (IMM-SPPC) is proposed, which converts the relative motion of the electrically driven eccentric rotor and the vibration environment into a second-order periodic coefficient differential equation. Through the calculation of the equilibrium point of the second-order periodic coefficient differential equation and the study of its stability, the synchronization criterion and the stability criterion of the eccentric rotor and the vibration environment are deduced. The simulation results show the validity of the deduced synchronization criterion and stability criterion. The proposed IMM-SPPC provides a new way for studying vibration synchronization.
\end{abstract}

\section{Introduction}

Beginning with the phenomenon discovered by Huijgens [1], vibration synchronizations have drawn researchers' attention in wide fields of mathematics, physics, and biology as well as in industrial applications of mechanical and electrical engineering, such as large-scale vibrating screen [2], vibrating feeder [3], and so on. There are two types of synchronizations in a general definition. The synchronous regime achieved by introducing special actions or imposing special constraints is defined as forced or controlled synchronization. The synchronous regime arose due to natural properties, and interactions of the processes are defined as self-synchronization, e.g., frequency synchronization of oscillating or rotating bodies. This paper focuses on addressing the problem of vibration synchronizations.

During the last decades, experts and scholars have made efforts on the vibration synchronization of mechanical systems and proposed three main methods such as the direct motion separation method, average torque difference method, and small parameter method [4-17]. The direct motion separation method was employed to address the vibration synchronization problem of objects with almost uniform rotations [9]. By using this method, the average speed and the phase difference of motors are formulated into fast motion component and slow motion component in the initial differential equation of the system motion. Then, the vibration synchronization problem is transformed into the solution existence of an integral stability criterion [7-9]. However, this method neglects the working conditions of the vibration systems [10] which results in certain limitations in practical engineering applications. In order to deal with this drawback, Wen proposed a principle of vibration synchronization of mechanical systems (vibratory synchronization transmission) by utilizing the average torque difference method, which transforms the vibratory motion into the phase difference of two eccentric rotors and introduces its corresponding working conditions $[6,11-13]$. Moreover, this method was applied to a plane-motion dualmass dual-machine offset self-synchronizing vibrator $[6,11]$, a plane single-mass dual-machine self-synchronizing vibrator with the same direction and reverse rotation [12], and a plane-motion single-mass dual-machine offset self-synchronizing vibrator [13]. However, the existing disturbances of average speed and phase difference of the two rotors are ignored. A modified average torque method with small 
parameters was introduced to convert the self-synchronization of two eccentric rotors into existence and stability of the zero solutions of the differential equations, including the disturbance parameters of phase difference and average rotational velocity [14-16]. Various mechanical systems are designed by employing this average torque method with small parameters, such as a dual-mass four-machine driven vibration system [14], an identical rotary two-machine driven vibration system with an unbalanced rotor [15], two nonidentical exciters driven nonresonant vibrating system [16], two cylindrical rollers in far resonance [17], the asymmetrically placed dual-machine drive rotation ultradistant resonance vibration system [18], two exciters driven dual-mass vibrating system [19], and frequency doubling control synchronization systems with two rotors in reverse rotation [20]. Although the mentioned modified average method is able to address the self-synchronization and controlled synchronization of two or more motors, the synchronization and stability criteria deviate from the perspective of vibratory synchronization transmission. However, the self-synchronization phenomena, such as vibratory synchronization between the moon's orbital motion and its rotational motion, have not been fully considered yet.

Motivated by the above discussion, this paper proposes a novel solution to address the self-synchronization problem of vibration systems. Assuming that an electrically driven eccentric rotor rotates anti-clockwise in a vibration environment. The vibration environment is set as a vibration field that changes periodically in $x$-direction. By defining a small parameter integral mean variable, the rotation motion of electrically driven eccentric rotor is converted into a second-order periodic coefficient differential equation. Then, the synchronization and stability criteria for the eccentric rotor and vibration environment are derived.

The contribution of this article is mainly reflected in the following aspects:

(1) An integral mean method with small parameters and periodic coefficient (IMM-SPPC) is proposed, which converts the relative motion of the eccentric rotor and the vibration environment into a second-order periodic coefficient differential equation.

(2) By solving the steady-state solution of the periodic coefficient differential equation at the equilibrium point, the synchronization criterion and the stability criterion of the eccentric rotor and the vibration environment are derived.

(3) The simulation results verify the validity of the synchronization criterion and the stability criterion and provide a novel way of studying vibration selfsynchronization.

This article gives a single-motor vibration model and establishes the differential equation of motion in the Section 2. Section 3 gives the synchronization and stability criteria of the single motor and the vibration environment. Section 4 carries out a computer simulation and verification of the criterion. Finally, some conclusions and discussions are drawn.

\section{Vibration System Modeling}

The dynamic model of the vibration system is shown in Figure 1. This system is composed of a motor and vibration environment. The motor has an eccentric rotor that rotates anti-clockwise. The vibration environment is the vibration field that changes periodically in $x$-direction. Assuming that there is a vibrating environment which exerts a vibration in the $x$-direction with $V_{x}=-\omega^{2} A \cos \omega t$, a rotor with an eccentric mass of $m$, an eccentric radius of $r$, and rotational inertia of $J$ rotates anti-clockwise around point $O$. Point $O$ satisfies the law of vibration motion with amplitude $A$.

The equation of motion for a vibrating environment is given by

$$
\left\{\begin{array}{l}
x=A \cos \varphi, \\
x^{\prime}=-\omega A \sin \varphi, \\
x^{\prime \prime}=-\omega^{2} A \cos \varphi,
\end{array}\right.
$$

where $x, x^{\prime}$, and $x^{\prime \prime}$ are the displacement, velocity, and acceleration of the vibrating environment, respectively, $\varphi$ is the angular displacement of the vibrating environment, $\omega$ is the rotating angular frequency of the vibrating environment, and $A$ is the amplitude of the vibrating environment.

2.1. Dynamic Equations of the Vibration System. Through the force analysis of the vibration system, the torque balance equation of the motor with eccentric rotor rotating in vibrating environment is obtained as follows:

$$
T_{J}+T_{f}+T_{d}=T_{M}+T_{z} .
$$

The dynamic equation of the eccentric rotor can be obtained as follows:

$$
T_{J}+T_{f}+T_{d}=T_{M}-\frac{1}{2} m r \omega^{2} A[\sin (\theta+\varphi)+\sin (\theta-\varphi)],
$$

where $T_{J}=J \theta^{\prime \prime}$ is the inertia torque of the eccentric rotor; $T_{f}$ is the moment of inertia of the vibration system; $T_{d}$ is the fan load of the excited motor; $T_{M}$ is the electromagnetic torque of the motor; $T_{z}=m r x^{\prime \prime} \sin \theta=-m r \omega^{2} A \cos \varphi \sin \theta$ is the vibration torque; and $\theta$ is the angular displacement of the eccentric rotor.

2.2. Differential Equations for the Relative Motion of the Vibration System. Since there is a rotational motion of the motor and the vibration of the vibration environment in a vibrating system, there is an interaction between the two motions, and this paper uses the vibration of the vibrating environment as a benchmark. Therefore, the mutual motion is studied within one cycle of the vibrating environment.

When the electrically driven eccentric rotor is captured by the same frequency of the vibration environment, the following conclusions can be drawn. 


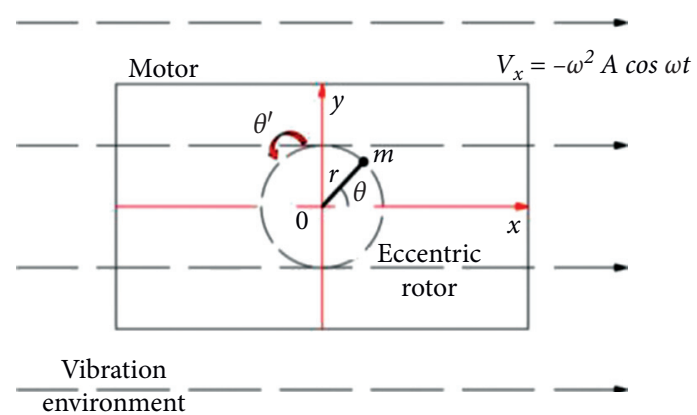

Figure 1: Dynamic model of the vibration system with electrically driven eccentric rotor in the vibration environment.

Assumption 1. The angular velocity of the eccentric rotor $\theta^{\prime}$ is captured by the angular frequency of the vibrating environment $\omega, \theta^{\prime}=\omega$.

Assumption 2. The difference between the angular displacement of the eccentric rotor $\theta$ and the angular displacement of the vibration $\varphi$ is $\Delta \alpha$, $(\omega / 2 \pi) \int_{t}^{t+(2 \pi / \omega)}(\theta-\varphi) \mathrm{d} t=\Delta \alpha$, where $\Delta \alpha$ is a constant and can be converted to the range of $[0,2 \pi]$.

Assumption 3. The angular velocity of the eccentric rotor $\theta^{\prime}$ and the angular velocity of the vibrating environment $\omega$ are equal $\operatorname{in}_{\text {nat }}$ the integration period $[t, t+(2 \pi / \omega)]$, $\omega / 2 \pi \int_{t}^{t+(2 \pi / \omega)}\left(\theta^{\prime}-\varphi^{\prime}\right) \mathrm{d} t=0$.

The small parameter integral mean variable of the angular displacement difference between the eccentric rotor and the vibrating environment is expressed as follows:

$$
\bar{\varepsilon}(t)=\frac{\omega}{2 \pi} \int_{t}^{t+(2 \pi / \omega)}(\theta-\varphi) \mathrm{d} t-\Delta \alpha,
$$

and then

$$
\begin{aligned}
\bar{\varepsilon}^{\prime}(t) & =\frac{\omega}{2 \pi} \int_{t}^{t+(2 \pi / \omega)}\left(\theta^{\prime}-\varphi^{\prime}\right) \mathrm{d} t, \\
\bar{\varepsilon}^{\prime \prime}(t) & =\frac{\omega}{2 \pi} \int_{t}^{t+(2 \pi / \omega)} \theta^{\prime \prime} \mathrm{d} t .
\end{aligned}
$$

When the vibration system is in stable synchronization, $\omega / 2 \pi \int_{t}^{t+(2 \pi / \omega)} \bar{\varepsilon}(t) \mathrm{d} t=0, \bar{\varepsilon}(t) \ll 1$.
At the torque balance point, we have

$$
\begin{aligned}
T_{M} & \approx T_{M 0}+C_{M}\left(\theta^{\prime}-\omega\right), \\
T_{d} & \approx T_{d 0}+m f d r \omega\left(\theta^{\prime}-\omega\right), \\
T_{f} & \approx T_{f 0}+f\left(\theta^{\prime}-\omega\right),
\end{aligned}
$$

and then

$$
\begin{aligned}
& C_{M}=\left.2 \pi \frac{\mathrm{d} T_{M}}{\mathrm{~d} \theta^{\prime}}\right|_{\omega}, \\
& T_{f 0}=f \omega, \\
& T_{d 0}=\frac{1}{2} m r f d \omega^{2},
\end{aligned}
$$

where $C_{M}$ is the mechanical characteristic slope when the electrically driven eccentric rotor is captured by the vibration environment, $T_{M O}$ is the electromagnetic torque when the electrically driven eccentric rotor is captured by the vibration environment, $T_{d o}$ is the fan load when the electrically driven eccentric rotor is captured by the vibration environment, $T_{f 0}$ is the friction torque when the eccentric rotor is captured by the vibration environment, $f$ is the friction coefficient of the vibration system, and $d$ is the equivalent diameter of the fan load of the motor.

This paper uses $\bar{\varepsilon}(t)$ as a variable and integrates equation (3) in $[t, t+(2 \pi / \omega)]$; we get

$$
\begin{aligned}
& \bar{\varepsilon}^{\prime \prime}(t)+\frac{f+m f d r \omega-C_{M}}{J} \bar{\varepsilon}^{\prime}(t)+\frac{T_{f 0}+T_{d 0}-T_{M 0}}{J} \\
& +\frac{m r \omega^{2} A}{2 J}\left[\frac{\omega}{2 \pi} \int_{t}^{t+(2 \pi / \omega)} \sin (\theta+\varphi) \mathrm{d} t+\frac{\omega}{2 \pi} \int_{t}^{t+(2 \pi / \omega)} \sin (\theta-\varphi) \mathrm{d} t\right]=0 .
\end{aligned}
$$

This section uses the torque balance equation to get the dynamic equation. Then, the dynamic differential equation is obtained by the differential mean method with small parameter periodic coefficients. Finally, a mathematical model of a single excited motor in a vibration environment is established, which provides a basis for the derivation of the 
synchronization and stability criterion of the vibration system.

\section{Synchronization Criterion and Stability Criterion of Vibration System}

3.1. Linearization of the Equation. In this paper, we study the relationship between $\theta-\varphi=\Delta \alpha$, so we linearize the equation at this point.

(1) $\omega / 2 \pi \int_{t}^{t+(2 \pi / \omega)} \sin (\theta-\varphi) \mathrm{d} t$ of linearization.

Expanding $\sin (\theta-\varphi)$ at $\theta-\varphi=\Delta \alpha$ by using Taylor formula and ignoring the higher term, it can be rewritten as

$$
\sin (\theta-\varphi)=\sin \Delta \alpha+(\theta-\varphi-\Delta \alpha) \cos \Delta \alpha .
$$

Integrating both sides of equation (10), we obtain

$$
\begin{aligned}
& \frac{\omega}{2 \pi} \int_{t}^{t+(2 \pi / \omega)} \sin (\theta-\varphi) \mathrm{d} t=\frac{\omega}{2 \pi} \\
& \cos \Delta \alpha \int_{t}^{t+(2 \pi / \omega)}(\theta-\varphi-\Delta \alpha) \mathrm{d} t+\sin \Delta \alpha .
\end{aligned}
$$

From equation (4),

$$
\frac{\omega}{2 \pi} \int_{t}^{t+(2 \pi / \omega)}(\theta-\varphi-\Delta \alpha) \mathrm{d} t=\bar{\varepsilon}(t) .
$$

Substituting equation (12) into equation (11), we can obtain

$\frac{\omega}{2 \pi} \int_{t}^{t+(2 \pi / \omega)} \sin (\theta-\varphi) \mathrm{d} t=\sin \Delta \alpha+\bar{\varepsilon}(t) \cos \Delta \alpha$.

(2) $\omega / 2 \pi \int_{t}^{t+(2 \pi / \omega)} \sin (\theta+\varphi) \mathrm{d} t$ of linearization.

Expanding $\sin (\theta+\varphi)$ at $\theta-\varphi=\Delta \alpha$ by using Taylor formula and ignoring the higher term, it can be rewritten as

$\sin (\theta+\varphi)=\sin (\Delta \alpha+2 \varphi)+(\theta-\varphi-\Delta \alpha) \cos (\Delta \alpha+2 \varphi)$.
Integrating both sides of equation (14), we obtain

$$
\begin{aligned}
& \frac{\omega}{2 \pi} \int_{t}^{t+(2 \pi / \omega)} \sin (\theta+\varphi) \mathrm{d} t \\
& =\frac{1}{2 \omega}\left[\left.\frac{\omega}{2 \pi}((\theta-\varphi-\Delta \alpha) \sin (\Delta \alpha+2 \varphi))\right|_{t} ^{t+(2 \pi / \omega)}\right] \\
& \quad-\frac{1}{2 \omega}\left[\frac{\omega}{2 \pi} \int_{t}^{t+(2 \pi / \omega)} \sin (\Delta \alpha+2 \varphi) \mathrm{d}(\theta-\varphi)\right] .
\end{aligned}
$$

From equation (5),

$\left.\frac{\omega}{2 \pi}[(\theta-\varphi-\Delta \alpha) \sin (\Delta \alpha+2 \varphi)]\right|_{t} ^{t+(2 \pi / \omega)}=\bar{\varepsilon}^{\prime}(t) \sin (\Delta \alpha+2 \varphi)$.

Substituting equation (16) into equation (15), we can obtain

$$
\begin{aligned}
\frac{\omega}{2 \pi} \int_{t}^{t+(2 \pi / \omega)} \sin (\theta+\varphi) \mathrm{d} t \\
=\frac{1}{2 \omega} \bar{\varepsilon} \prime(t) \sin (\Delta \alpha+2 \varphi)+\left(\frac{1}{2 \omega}\right)^{2} \\
\quad \cdot\left[\left.\frac{\omega}{2 \pi}\left(\left(\theta^{\prime}-\omega\right) \cos (\Delta \alpha+2 \varphi)\right)\right|_{t} ^{t+(2 \pi / \omega)}\right] \\
\quad-\left(\frac{1}{2 \omega}\right)^{2}\left[\frac{\omega}{2 \pi} \int_{t}^{t+(2 \pi / \omega)} \cos (\Delta \alpha+2 \varphi) \mathrm{d}\left(\theta \prime-\varphi^{\prime}\right)\right]
\end{aligned}
$$

From equation (6),

$$
\left.\frac{\omega}{2 \pi}\left[\left(\theta^{\prime}-\omega\right) \cos (\Delta \alpha+2 \varphi)\right]\right|_{t} ^{t+(2 \pi / \omega)}=\bar{\varepsilon}^{\prime \prime}(t) \cos (\Delta \alpha+2 \varphi) .
$$

Substituting equation (18) into equation (16), we can obtain

$$
\begin{aligned}
& \frac{\omega}{2 \pi} \int_{t}^{t+\frac{2 \pi}{\omega}} \sin (\theta+\varphi) d t=\frac{1}{2 \omega} \bar{\varepsilon}^{\prime}(t) \sin (\Delta \alpha+2 \varphi)+\left(\frac{1}{2 \omega}\right)^{2} \bar{\varepsilon}^{\prime \prime}(t) \cos (\Delta \alpha+2 \varphi) \\
& -\left(\frac{1}{2 \omega}\right)^{2}\left[\frac{\omega}{2 \pi} \int_{t}^{t+\frac{2 \pi}{\omega}} \cos (\Delta \alpha+2 \varphi) d\left(\theta^{\prime}-\varphi^{\prime}\right)\right]
\end{aligned}
$$

We can find that $\omega / 2 \pi \int_{t}^{t+(2 \pi / \omega)} \sin (\theta+\varphi) \mathrm{d} t$ is an infinite-order function of $\bar{\varepsilon}^{\prime}(t), \bar{\varepsilon}^{\prime \prime}(t), \ldots \ldots$ and ignoring the higher-order differential terms, we can obtain 


$$
\frac{\omega}{2 \pi} \int_{t}^{t+(2 \pi / \omega)} \sin (\theta+\varphi) \mathrm{d} t \approx \frac{1}{2 \omega} \bar{\varepsilon}^{\prime}(t) \sin (\Delta \alpha+2 \varphi)+\left(\frac{1}{2 \omega}\right)^{2} \bar{\varepsilon}^{\prime \prime}(t) \cos (\Delta \alpha+2 \varphi) .
$$

The small parameter integral mean periodic coefficient differential equation of an eccentric rotor in vibration environment can be obtained as follows:

$$
\begin{gathered}
\frac{8 J+m r A \cos (\Delta \alpha+2 \varphi)}{8 J} \bar{\varepsilon}^{\prime \prime}(t)+\frac{4\left(f+m f d r \omega-C_{M}\right)+m r \omega A \sin (\Delta \alpha+2 \varphi)}{4 J} \bar{\varepsilon}^{\prime}(t) \\
+\frac{m r \omega^{2} A \cos \Delta \alpha}{2 J} \bar{\varepsilon}(t)+\frac{m r \omega^{2} A \sin \Delta \alpha}{2 J}-\frac{T_{M 0}-T_{f 0}-T_{d 0}}{J}=0 .
\end{gathered}
$$

Finally, we obtain differential equation (21), which makes it possible to better meet the synchronization and stability conditions.

3.2. Synchronization Criterion of Vibration System. When the eccentric rotor is synchronized with the vibration environment,

$$
\bar{\varepsilon}^{\prime \prime}(t)=\bar{\varepsilon}^{\prime}(t)=\bar{\varepsilon}(t)=0 .
$$

Substituting equation (22) into equation (21), we can obtain

$$
-\frac{m r \omega^{2} A}{2 J} \sin \Delta \alpha+\frac{T_{M 0}-T_{f 0}-T_{d 0}}{J}=0,
$$

and then

$$
\sin \Delta \alpha=\frac{2\left(T_{M 0}-T_{f 0}-T_{d 0}\right)}{m r \omega^{2} A} .
$$

The synchronization criterion can be obtained as follows:

$$
\left|\frac{2\left(T_{M 0}-T_{f 0}-T_{d 0}\right)}{m r \omega^{2} A}\right|<1 .
$$

When the angular frequency of the vibration environment is less than the no-load angular velocity of motor $\omega_{0}$, the motor is running in the electric working state.
From equation (24),

$$
\begin{aligned}
\left(T_{M 0}-T_{f 0}-T_{d 0}\right) & >0 \sin \Delta \alpha>0, \\
\bar{T}_{z 0} & =\frac{m r \omega^{2} A}{2} \sin \Delta \alpha>0 .
\end{aligned}
$$

The vibration torque is a motor load torque with $\bar{T}_{z 0}$ as the average value. And the synchronization phase difference $\Delta \alpha$ can be located in the first or second quadrant.

When the angular frequency of the vibration environment is greater than the no-load angular velocity of motor $\omega_{0}$, the motor is running in the feedback state.

$$
\begin{aligned}
\left(T_{M 0}-T_{f 0}-T_{d 0}\right) & >0 \sin \Delta \alpha>0, \\
\bar{T}_{z 0} & =\frac{m r \omega^{2} A}{2} \sin \Delta \alpha>0 .
\end{aligned}
$$

The vibration torque is an active torque with $\bar{T}_{z 0}$ as the average value. The phase difference $\Delta \alpha$ can be located in the third or fourth quadrant.

3.3. Stability Criterion of Vibration System. The periodic coefficient differential equation of eccentric rotor frequency being captured by vibration environment can be transferred like this.

$$
\bar{\varepsilon}^{\prime \prime}(t)+\frac{8\left(f+m f d r \omega-C_{M}\right)+2 m r \omega A \sin (\Delta \alpha+2 \varphi)}{8 J+m r A \cos (\Delta \alpha+2 \varphi)} \bar{\varepsilon}^{\prime}(t)+\frac{4 m r \omega^{2} A \cos \Delta \alpha}{8 J+m r A \cos (\Delta \alpha+2 \varphi)} \bar{\varepsilon}(t)=0 .
$$

Equation (28) is a second-order periodic coefficient linear differential equation with a period of $\pi / \omega$. According to the theory of second-order differential equations [21, 22], if equation (28) is to reach stable state, the two conditions must be met.

$$
\left\{\begin{array}{l}
\frac{4 m r \omega^{2} A}{8 J+m r A \cos (\Delta \alpha+2 \varphi)} \cos \Delta \alpha>0, \\
\int_{0}^{\pi / \omega} \frac{8\left(f+m f d r \omega-C_{M}\right)+2 m r \omega A \sin (\Delta \alpha+2 \varphi)}{8 J+m r A \cos (\Delta \alpha+2 \varphi)} \mathrm{d} t>0 .
\end{array}\right.
$$


Commonly, those conditions are stratified, $J>m r A$.

From the first condition, we obtain

$$
\cos \Delta \alpha>0 \text {. }
$$

From the second condition, we obtain

$$
\begin{aligned}
& \int_{0}^{\pi / \omega} \frac{8\left(f+m f d r \omega-C_{M}\right)+2 m r \omega A \sin (\Delta \alpha+2 \varphi)}{8 J+m r A \cos (\Delta \alpha+2 \varphi)} d t \\
& =\frac{4 \pi\left(f+m f d r \omega-C_{M}\right)}{\omega \sqrt{64 J^{2}-m^{2} r^{2} A^{2}}}>0 .
\end{aligned}
$$

The stability criterion can be obtained as follows:

$$
\cos \Delta \alpha>0
$$

Remark 1 . The synchronization criterion and stability criterion can estimate the quadrant range of the synchronization phase difference $\Delta \alpha$.

When the angular frequency of the vibration environment is less than the no-load angular velocity of motor $\omega_{0}$,

$$
\left\{\begin{array}{l}
\sin \Delta \alpha>0, \\
\cos \Delta \alpha>0 .
\end{array}\right.
$$

The motor is running in the electric working state. The phase difference $\Delta \alpha$ can be located in the first quadrant.

When the angular frequency of the vibration environment is greater than the no-load angular velocity of motor $\omega_{0}$,

$$
\left\{\begin{array}{l}
\sin \Delta \alpha<0, \\
\cos \Delta \alpha>0
\end{array}\right.
$$

The motor is running in the feedback state. The phase difference $\Delta \alpha$ can be located in the fourth quadrant.

\section{Simulation Results and Discussion}

In this section, some quantitative analyses are given to illustrate the effectiveness of the aforementioned theoretical results.

4.1. Setting Parameters. The motor model is established based on the practical expression of the mechanical characteristics of the asynchronous motor. The model parameters are listed in Table 1.

4.2. Simulation and Analysis. Based on the established mathematical model, a simulation model is established in MATLAB/Simulink.

Simulation 1: the angular velocity is $\omega=24.9 \pi \mathrm{rad} / \mathrm{s}$.

When $\omega=24.9 \pi \mathrm{rad} / \mathrm{s}$, we get slip rate $s=0.004$, $T_{M 0}=7.949, \quad T_{f_{0}}=f \omega \approx 0.156, \quad T_{d_{0}}=m r \omega^{2} f d / 2$
TABle 1: Parameters of the vibration system.

\begin{tabular}{lc}
\hline Parameters & Value \\
\hline Maximum torque of motor $T_{M}(\mathrm{~N} \cdot \mathrm{m})$ & 50 \\
Maximum slip of motor $S_{M}$ & 0.05 \\
No-load angular velocity of exciter $\omega_{0}(\mathrm{rad} / \mathrm{s})$ & $25 \pi$ \\
Amplitude of simple harmonic $A(\mathrm{~m})$ & 0.003 \\
Rotation radius of eccentric rotor $r(\mathrm{~m})$ & 0.2 \\
Mass of eccentric rotor $m(\mathrm{~kg})$ & 10 \\
Load equivalent diameter of motor fan $d(\mathrm{~m})$ & 0.1 \\
Coefficient of friction $f$ & 0.002 \\
Moment of inertia of eccentric rotor $J\left(\mathrm{~kg} \cdot \mathrm{m}^{2}\right)$ & 0.02 \\
Simulation time T $(\mathrm{s})$ & 100
\end{tabular}

$\approx 0.612$, and $m r \omega^{2} A=18.358$; substituting the above values into the synchronization criterion, we obtain

$$
\left|\frac{2\left(T_{M 0}-T_{f 0}-T_{d 0}\right)}{m r \omega^{2} A}\right| \approx 0.782<1 .
$$

The above values satisfy the criterion of synchronization, and the phase difference is in the first quadrant. The simulation results are shown in Figure 2.

The motor is in electric working state, and the synchronization phase difference $\Delta \alpha \approx-80.791 \mathrm{rad}$ (approximately $51.02^{\circ}$ in the range of $360^{\circ}$ ) is in the first quadrant.

Simulation 2: the angular velocity is $\omega=25.1 \pi \mathrm{rad} / \mathrm{s}$.

When $\omega=25.1 \pi \mathrm{rad} / \mathrm{s}$, we get slip rate $s=-0.004$, $T_{M 0}=-7.949, \quad T_{f_{0}}=f \omega \approx 0.158, \quad T_{d_{0}}=m r \omega^{2} f d / 2$ $\approx 0.622$, and $m r \omega^{2} A=18.654$; substituting the above values into the synchronization criterion, we obtain

$$
\left|\frac{2\left(T_{M 0}-T_{f 0}-T_{d 0}\right)}{m r \omega^{2} A}\right| \approx 0.935<1 .
$$

The above values satisfy the criterion of synchronization, and the phase difference is in the fourth quadrant. The simulation results are shown in Figure 3.

The motor is in the feedback state, and the synchronization phase difference $\Delta \alpha \approx-89.164 \mathrm{rad}$ (approximately $291.28^{\circ}$ in the range of $360^{\circ}$ ) is in the fourth quadrant.

Simulation 3: the angular velocity is $\omega=24.9 \pi \mathrm{rad} / \mathrm{s}$ and amplitude is $A=0.002 \mathrm{~m}$.

When $\omega=24.9 \pi \mathrm{rad} / \mathrm{s}$, we get slip rate $s=0.004$, $T_{M 0}=7.949, \quad T_{f_{0}}=f \omega \approx 0.156, \quad T_{d_{0}}=m r \omega^{2} f d / 2$ $\approx 0.612$, and $m r \omega^{2} A=12.239$; substituting the above values into the synchronization criterion, we obtain

$$
\left|\frac{2\left(T_{M 0}-T_{f 0}-T_{d 0}\right)}{m r \omega^{2} A}\right| \approx 1.17>1 .
$$

The above values do not satisfy the criterion of synchronization. The simulation results are shown in Figure 4. 


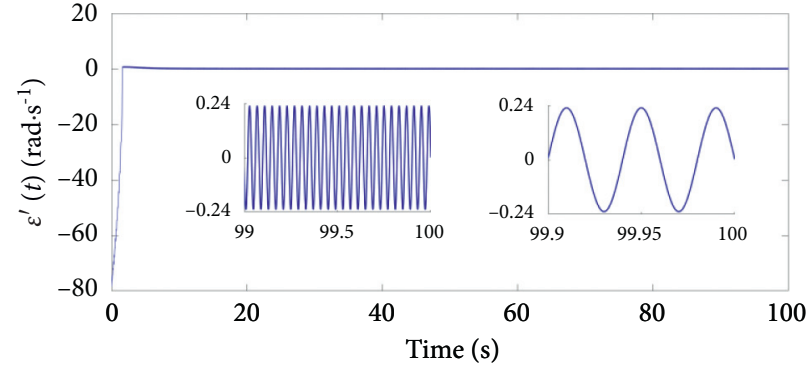

(a)

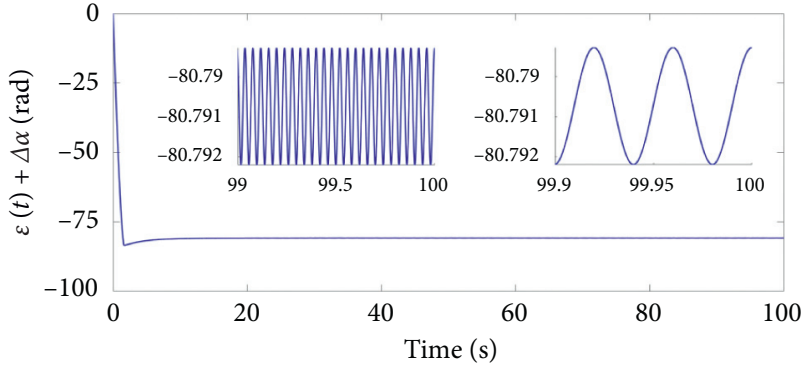

(b)

FIGURE 2: Simulation curve when $\omega=24.9 \pi$. (a) $\varepsilon^{\prime}(t)$ graph. (b) $\varepsilon(t)+\Delta \alpha$ graph.

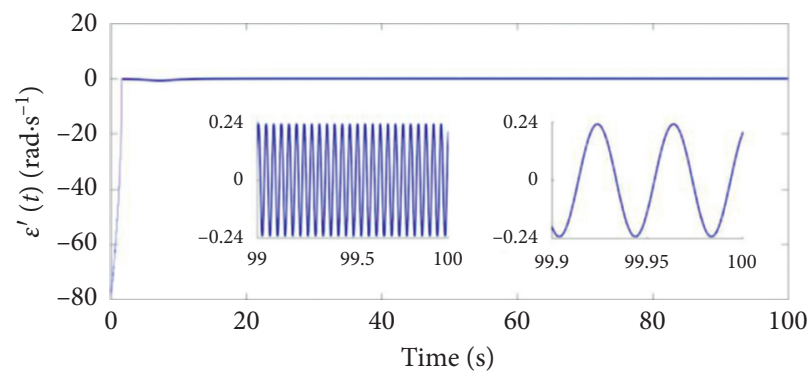

(a)

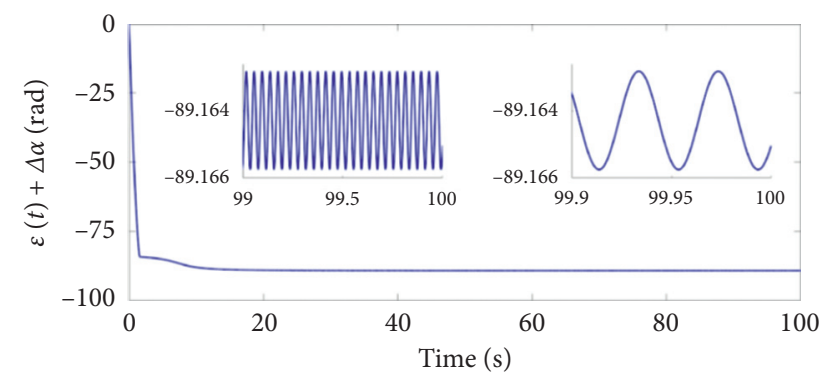

(b)

Figure 3: Simulation curve when $\omega=25.1 \pi$. (a) $\varepsilon^{\prime}(t)$ graph. (b) $\varepsilon(t)+\Delta \alpha$ graph.

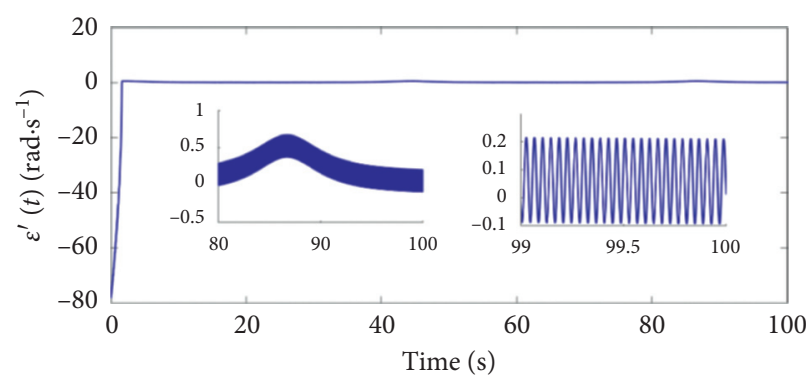

(a)

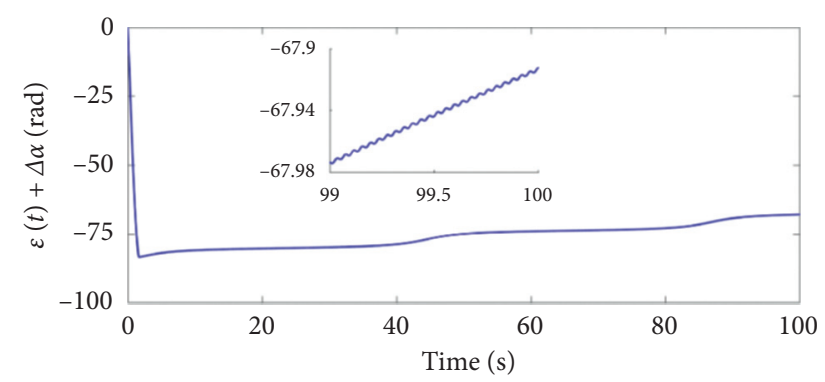

(b)

FIGURE 4: Simulation curve when $\omega=24.9 \pi$ and $A=0.002 \mathrm{~m}$. (a) $\varepsilon^{\prime}(t)$ graph. (b) $\varepsilon(t)+\Delta \alpha$ graph.

\section{Conclusions and Discussion}

5.1. Main Conclusions. In this paper, a new method for vibration synchronization research using small parameter integral mean periodic coefficient differential equation is proposed. The system is transformed into a second-order periodic coefficient differential equation so that the related theories of the second-order periodic coefficient differential equation can be used for synchronization and stability analysis.

The synchronization criterion and stability criterion of the vibration of the electrically driven eccentric rotor in a vibrating environment are obtained. The combination of the two criteria determines the stable phase of the electrically driven eccentric rotor in the vibrating environment. The idea of synchronization between the eccentric rotor and the vibration environment proposed in this paper provides a new way to study vibration synchronization. The method of differential equations with small parameter mean and variable periodic coefficients provides a new method for studying vibration synchronization.

5.2. Discussion. Since the periodic coefficient differential equation based on small parameter integral mean variable is used to research on vibration synchronization theory, the problem of vibration synchronization is transformed into a second-order periodic coefficient differential equation. In 
essence, it is still the basic idea of the averaging method, which also makes the influence of the moment of inertia not be reflected in the stability criterion. The authors will further carry out related research.

\section{Data Availability}

The results is based on the theory calculation, and it is also verified by simulation with Matlab SIMULATION. The authors can provide the simulation model upon request.

\section{Conflicts of Interest}

The authors declare that they have no conflicts of interest.

\section{Acknowledgments}

This research was financially supported by the Special Fund of Chinese Central Government Guidance for Local Science and Technology Development (grant no. [2016] 4006). Moreover, this research was also financially supported by the Engineering Machinery Energy-Saving Transmission and Control Innovation Team of Guizhou (grant no. [2014] 4013). The authors also thank CINTEC Heavy Equipment (China) Co., Ltd., for the support.

\section{References}

[1] C. Huijgens, Sive de Motu Pendulorum ad Horologia Aptato Demonstrationes Geometricae, Horoloqium Oscilatorium, Paris, France, 1673.

[2] J. Jiang, C. Chen, and Bo Lei, "Controlled synchronization of vibrating screen driven by two exciters rotating in the opposite direction," Journal of Vibration and Shock, vol. 38, no. 8, pp. 126-132, 2019.

[3] X. Kang, The Research of Self-Synchronous Vibrating Fixed Quantity Feeding System, Northeastern University, Boston, MA, USA, 2008.

[4] I. I. Blekhman, A. L. Fradkov, H. Nijmeijer, and A. Y. Pogromsky, "On self-synchronization and controlled synchronization," Systems \& Control Letters, vol. 31, no. 5, pp. 299-305, 1997.

[5] I. I. Blekhman, "Self-synchronization of vibrators in some types of vibration machines," Inzhenerny Sbornik, vol. 16, pp. 49-72, 1953

[6] B. Wen, "Some new results to research on "vibtation synchronizatioin theory" with its application," Journal of Vibration and Shock, vol. 3, pp. 1-10, 1983.

[7] I. I. Blekhman, Vibrational Mechanics, World Scientific Publishing, Singapore, 2000.

[8] I. I. Blekhman, Selected Topics in Vibrational Mechanics, World Scientific Publishing, Singapore, 2004.

[9] I. I. Blekhman and N. P. Yaroshevich, "Extension of the domain of applicability of the integral stability criterion (extremum property) in synchronization problems," Journal of Applied Mathematics and Mechanics, vol. 68, no. 6, pp. 839-846, 2004.

[10] X. Zhang, Study on Synchronization Theory in the Vibrating System Driven by Double-Motor and Multi-Motor, Northeastern University, Shenyang, China, 2014.

[11] B. Wen and X. Lin, "Vibration synchronous transmission and its industrial application," Journal of Mechanical Engineering, vol. 20, no. 3, pp. 26-42, 1984.
[12] B. Wen and L. Guan, "Synchronization theory and debugging method of self-synchronization vibration machine," Mining \& Processing Equipment, vol. 5, pp. 35-45, 1979.

[13] B. Wen, "Research on the movement law of the offset selfsynchronizing vibrator," Chinese Journal of Applied Mechanics, vol. 3, pp. 23-36, 1985.

[14] C. Zhao, Q. Zhao, and Z. Gong, "Synchronization of two selfsynchronous vibrating machines on an isolation frame," Shock and Vibration, vol. 18, no. 1-2, pp. 73-90, 2011.

[15] C. Zhao, Q. Zhao, Y. Zhang, and B. Wen, "Synchronization of two non-identical coupled exciters in a non-resonant vibrating system of plane motion," Journal of Mechanical Science and Technology, vol. 25, no. 1, pp. 49-60, 2011.

[16] X. Zhang, C. Zhao, and B. Wen, "Theoretical and experimental study on synchronization of the two homodromy exciters in a non-resonant vibrating system," Shock and Vibration, vol. 20, no. 2, pp. 327-340, 2012.

[17] D. Gu, B. Wen, and J. Zhang, "Vibratory synchronization transmission of two cylindrical rollers in a super-resonant vibrating system with symmetrical structure," Proceedings of the Institution of Mechanical Engineers, Part C: Journal of Mechanical Engineering Science, vol. 233, no. 4, pp. 12041223, 2019.

[18] D. Gu, Y. Liu, and J. Zhang, "Asymmetric double vibration exciter vibration synchronous transmission," Journal of Vibration and Shock, vol. 38, no. 1, pp. 37-43, 2019.

[19] Y. Liu, X. Zhang, and D. Gu, "Synchronization of a dual-mass vibrating system with two exciters," Shock and Vibration, vol. 2020, Article ID 9345652, 2020.

[20] Y. Liu, L. Jia, and B. Wen, "Synchronization of frequency multiplier control of vibration system driven by two machines," Journal of Northeastern University (Natural Science), vol. 40, no. 12, pp. 1726-1731, 2019.

[21] J. L. Shi, "On stability of two order linear differential equations with periodic coefficient," Acta Mathematica Scientia, vol. 20, no. 1, pp. 130-139, 2000.

[22] X. Chen, "Characteristic exponent for second order periodic linear differential equations," Annals of Differential Equation, vol. 13, pp. 107-118, 1997. 